\title{
Cobra head sign of adult-type ureterocele incidentally discovered during pre-operative evaluation for fibroid uterus before abdominal hysterectomy
}

\author{
Ibrahim Abdelazim ${ }^{1,2}$, Mohannad Abu-Faza² \\ 'Department of Obstetrics and Gynecology, Ain Shams University, Cairo, Egypt \\ 2Department of Obstetrics and Gynecology, Ahmadi Hospital, Kuwait Oil Company, Ahmadi, Kuwait
}

\begin{abstract}
Corresponding author: Ibrahim Abdelazim, Ahmadi hospital, Kuwait Oil Compnay (KOC), Ahmadi, Kuwait, phone: $+965-66551300$, e-mail: dr.ibrahimanwar@gmail.com
\end{abstract}

The female genital and urinary tracts are closely related, and there is potential risk of injury to one when operating on the other [1].

The risk of damage increases when the normal anatomy is altered by primary pathology or adhesions or when the normal anatomy is insufficiently identified intraoperatively during severe bleeding [1].

There are certain conditions that increase the risk of ureteral injuries as endometriosis, large ovarian masses, pelvic inflammatory disease (PID), pelvic malignancy, irradiation, adhesions, previous pelvic surgery, and broad ligament fibroids $[2,3]$.

Urinary tract injury complicates $0.2-1 \%$ of all gynaecological and pelvic operations [4]. Eighty-two percent of ureter injuries occur during pelvic surgery, and $75 \%$ of urinary tract injuries are due to gynaecological surgery [4].

Previous studies have recommended proper ureteric identification before hysterectomy for women with increased risk of ureteric injuries [2,3].

This report represents a case report of multiple fibroid uterus discovered to have a left uncomplicated ureterocele, to highlight the value of following hospital policies and/or protocols, and the value of a multidisciplinary team approach in the management of such cases.

In addition, the report highlights the value of ureteric identification either pre-operatively using the intravenous pyelography or intraoperatively using the direct ureteric identification, and/or ureteric catheterization for women at risk of ureteric injuries, to avoid such problems during surgery.

A 49-year-old woman presented to the Gynaecology outpatients' clinic of Ahmadi hospital, Kuwait, with menorrhagia over the last year due to multiple fibroid uterus diagnosed by magnetic resonance imaging (MRI) (Figure 1).

The studied woman signed a written consent form for abdominal hysterectomy, and the pre-operative investigations done for the studied woman according to the hospital protocol including the following: complete blood picture, liver, and kidney function tests, prothrombin time, and pre-operative intravenous pyelography, showed cobra head sign (spring onion sign) of the left ureter.

Cobra head sign refers to dilatation of the distal ureter, surrounded by thin lucent line, which can be seen in adulttype uncomplicated ureterocele (Figure 2).

The lucent "hood" of the cobra represents the combined thickness of the ureteral wall and prolapsed bladder mucosa outlined by contrast material within the bladder lumen (Figure 2).

Intra-operative cystoscopy was performed for the studied woman for ureteric catheterisation and proper ureteric identification to avoid intra-operative ureteric injury during abdominal hysterectomy according to the hospital protocol. 


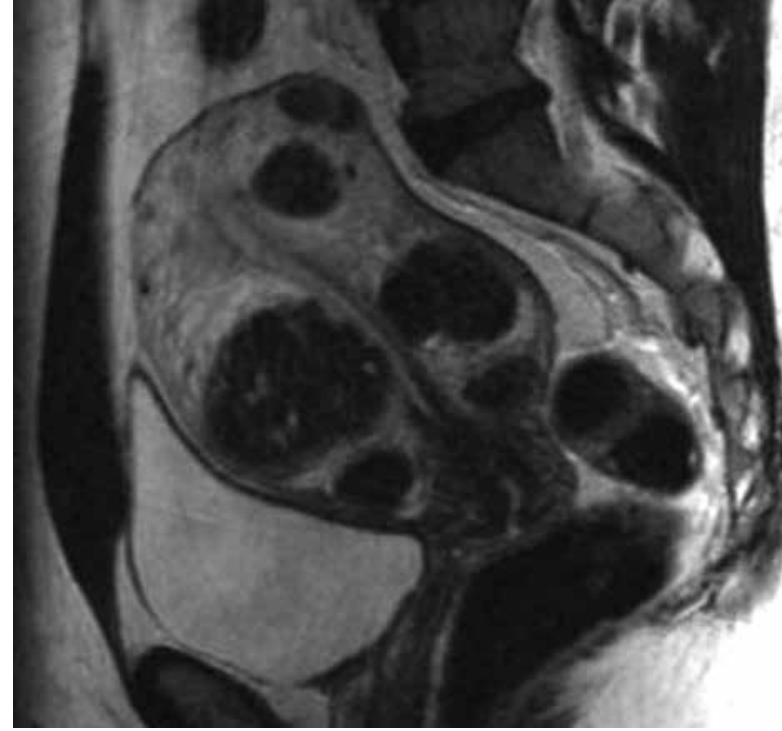

Figure 1. Magnetic resonance image (MRI) shows multiple fibroid uterus

The cystoscopy confirmed the cystic dilation of the terminal part of the left ureter (left uncomplicated ureterocele).

The ureteric catheters were inserted on the left side with uncomplicated ureterocele (Figure 3), and on the right normal side (Figure 4) before abdominal hysterectomy by the urology consultant, and they were removed immediately after the hysterectomy.

The studied woman had smooth post-operative recovery after the abdominal hysterectomy and was discharged from the hospital in good general condition for follow-up in the urology outpatient clinic.

Written consent and local Ethics Committee approval were obtained to present the studied woman's case as a case report.

Ureterocele is defined as a cystic dilatation of distal ureter associated tissue defect in the bladder, neck of the bladder, or even extending into the posterior urethra. The incidence of ureterocele is estimated to be 1 in 4000 live births [5].

The use of ultrasound allowed the antenatal diagnosis of asymptomatic ureterocele [5]. Recurrent urinary tract in-

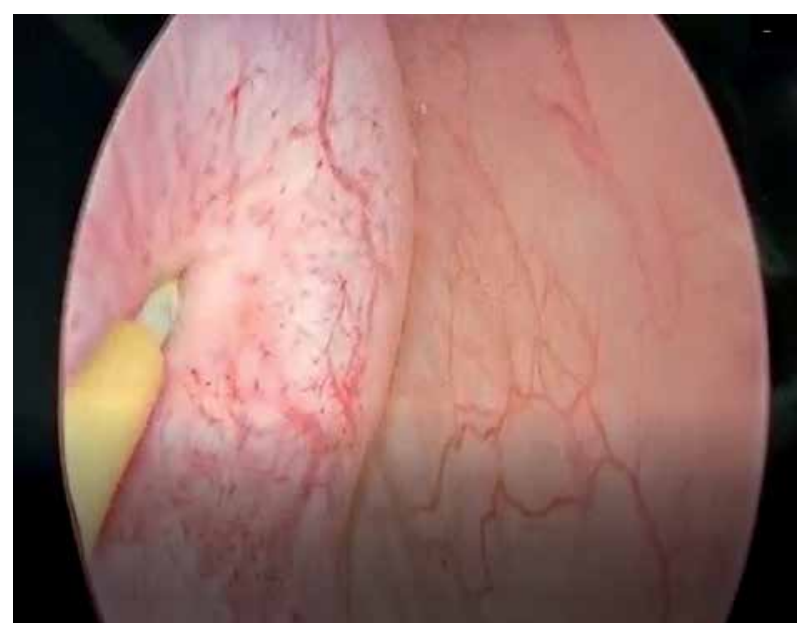

Figure 3. Cystoscopy and intra-operative ureteric catheterisation of the left uncomplicated ureterocele

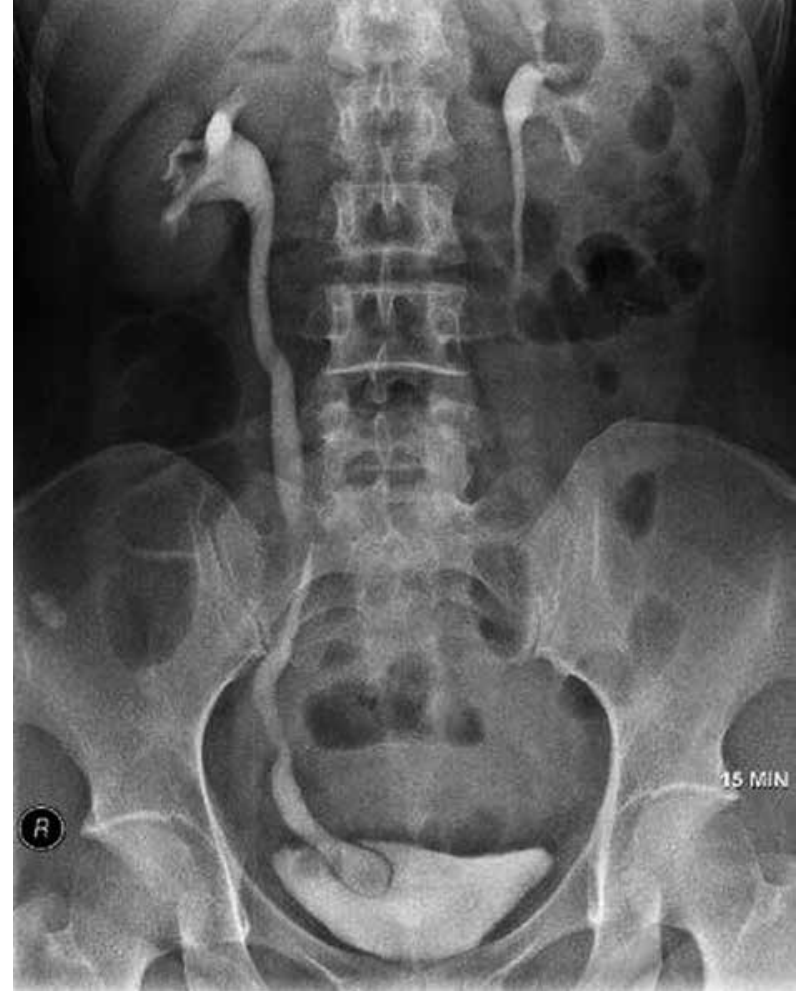

Figure 2. Intravenous pyelography shows the cobra head sign of the left uncomplicated ureterocele

fection is the most common symptom of ureterocele after birth [6].

Intravenous pyelogram, isotope renogram, magnetic resonance imaging, and cystoscopy can be used for complete understanding of the ureterocele, associated anatomical defects in the bladder, and/or posterior urethra $[7,8]$.

The old, traditional, aggressive approach in the management of ureterocele changed to conservative management in the last few years, and asymptomatic ureteroceles discovered accidentally on ultrasound or intravenous pyelography (IVP) with normal drainage can be left without surgical intervention (conservative management and follow-up) $[5,9]$.

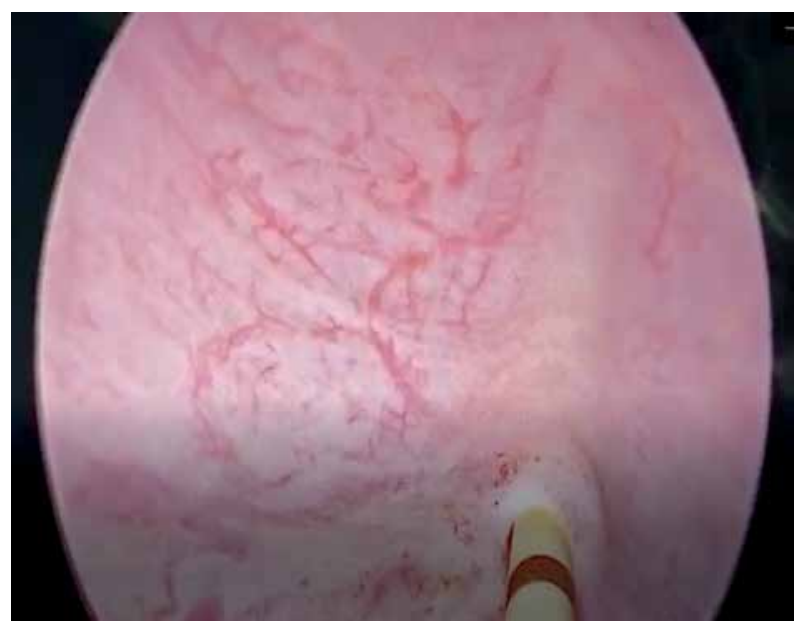

Figure 4. Ureteric catheterisation of the normal right ureter 
This case report of multiple fibroid uterus discovered to have left uncomplicated ureterocele is presented to highlight the value of following hospital policies and/or protocols and the value of a multidisciplinary team approach in the management of such cases. In addition, it highlights the value of ureteric identification either preoperatively using the intravenous pyelography or intraoperatively using the direct ureteric identification and/or ureteric catheterization for women at risk of ureteric injuries, to avoid such problems during surgery.

In conclusion, it is important to follow hospital policies and/or protocols, and to identify the ureteric anatomy preoperatively using intravenous pyelography or intraoperatively using direct ureteric catheterization for women at risk of ureteric injuries, to avoid such problems during surgery.

\section{Acknowledgments}

The authors are grateful to the studied woman who agreed and gave consent to participate in this report.

\section{Conflict of interest}

The authors declare no conflict of interest.

\section{References}

1. Ozdemir E, Ozturk U, Celen S, et al. Urinary complications of gynecologic surgery: iatrogenic urinary tract system injuries in obstetrics and gynecology operations. Clin Exp Obstet Gynecol 2011; 38: 217-20.

2. Chan JK, Morrow J, Manetta A. Prevention of ureteral injuries in gynecologic surgery. Am J Obstet Gynecol 2003; 188: 1273-7.

3. Lee Z, Kaplan J, Giusto L, Eun D. Prevention of iatrogenic ureteral injuries during robotic gynecologic surgery: a review. Am J Obstet Gynecol 2016; 214: 566-71.

4. Lee JS, Choe JH, Lee HS, Seo JT. Urologic complications following obstetric and gynecologic surgery. Korean J Urol 2012; 53: 795-9.

5. Chowdhary SK, Kandpal DK, Sibal A, Srivastava RN. Management of complicated ureteroceles: Different modalities of treatment and long-term outcome. J Indian Assoc Pediatr Surg 2014; 19: 156-61.

6. Hari P, Singla IK, Mantan M, Kanitkar M, Batra B, Bagga A. Chronic renal failure in children. Indian Pediatr 2003; 40: 1035-42.

7. Mohta A, Upreti L. Cecoureterocele. Indian Pediatr 2010; 47: 522-3.

8. Shokeir AA, Nijman RJ. Ureterocele: an ongoing challenge in infancy and childhood. BJU Int 2002; 90: 777-83.

9. Coplen DE, Barthold JS. Controversies in the management of ectopic ureteroceles. Urology 2000; 56: 665-8. 